\title{
Alfvén wave filamentation and dispersive phase mixing in a high-density channel: Landau fluid and hybrid simulations
}

\author{
D. Borgogno ${ }^{1}$, P. Hellinger ${ }^{2}$, T. Passot ${ }^{1}$, P. L. Sulem ${ }^{1}$, and P. M. Trávníček ${ }^{2}$ \\ ${ }^{1}$ Université de Nice Sophia Antipolis, CNRS, Observatoire de la Côte d'Azur, B.P. 4229, 06304 Nice Cedex 4, France \\ ${ }^{2}$ Institute of Atmospheric Physics and Astronomical Institute, AS CR Bocni II/1401, 14131 Prague, Czech Republic
}

Received: 9 December 2008 - Revised: 24 March 2009 - Accepted: 26 March 2009 - Published: 8 April 2009

\begin{abstract}
The propagation of dispersive Alfvén waves in a low-beta collisionless plasma with a high-density channel aligned with the ambient magnetic field, is studied in three space dimensions. A fluid model retaining linear Landau damping and finite Larmor radius corrections is used, together with a hybrid particle-in-cell simulation aimed to validate the predictions of this Landau-fluid model. It is shown that when the density enhancement is moderate (depending on the pump wavelength and the plasma parameters), the wave energy concentrates into a filament whose transverse size is prescribed by the dimension of the channel. In contrast, in the case of a stronger density perturbation, the early formation of a magnetic filament is followed by the onset of thin helical ribbons and the development of strong gradients. This "dispersive phase mixing" provides a mechanism permitting dissipation processes (not included in the present model) to act and heat the plasma.
\end{abstract}

\section{Introduction}

The phenomenon of Alfvén wave filamentation is viewed as one of the possible mechanisms at the origin of the magnetic tubes observed in the magnetosheath (Alexandrova et al., 2004, 2006). It is associated with the transverse instability of a small-amplitude quasi-monochromatic pump Alfvén wave propagating along an ambient magnetic field, in conditions where dispersion is significant. The problem, initiated by Shukla and Stenflo (1989), was studied in detail in the framework of the magnetohydrodynamics with Hall effect (Hall-MHD) for a homogeneous plasma, using both asymptotic (Champeaux et al., 1997; Laveder et al., 2003) and numerical (Laveder et al., 2002; Dreher et al., 2005)

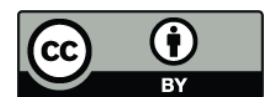

Correspondence to: P. L. Sulem (sulem@oca.eu) approaches. The former procedure consists mostly in a modulation analysis leading to a two-dimensional nonlinear Schrödinger (NLS) equation for the pump amplitude subject to purely transverse perturbations. Depending on the pump wavelength and the plasma parameters (a condition that reduces to $\beta>1$ in the long-wavelength limit), an instability can develop and become explosive in the nonlinear regime, being associated with the finite-time blowup of solutions of the two-dimensional NLS equation (Sulem and Sulem, 1999).

The question then arises of the influence of inhomogeneities that are usually present both in astrophysical and laboratory plasmas. A few studies have been devoted to the instabilities of dispersive Alfvén waves in the presence of inhomogeneities in the direction of propagation, making use of a long-wavelength weakly nonlinear asymptotics (Buti, 1991; Buti et al., 1999; Ruderman, 2002). In the absence of dispersion, transverse inhomogeneities are often considered as a main source of small-scale generation through the phenomenon of phase mixing that progressively distorts plane Alfvén fronts due to the variation of the Alfvén speed across the magnetic field. This results in the creation of strong gradients which, in a collisional plasma, enhance Joule dissipation (Heyvaerts and Priest, 1983; Ireland and Priest, 1997). Applications to the heating of the solar corona have been an important motivation for these studies. A problem then concerns the nonlinear development of phase mixing in a collisionless plasma where dispersive effects start to be effective at scales larger than those where dissipation sets in. The issue of the contribution of circularly polarized Alfvén waves to the heating of a collisionless magnetized plasma in the presence of a strong density enhancement and to the energy and momentum transport in such a medium, was recently addressed in the very low beta regime, using particle in cell (PIC) simulations in two space dimensions that retain the full microphysics while assuming, because of computational constraints, a reduced ion-electron mass ratio (Tsiklauri et al., 2005a,b; Mottez et al., 2006). While no electric

Published by Copernicus Publications on behalf of the European Geosciences Union and the American Geophysical Union. 
field is associated with transversally-homogeneous parallelpropagating Alfvén waves, a parallel electric field permitting particle acceleration along the ambient field develops when the waves are structured in the perpendicular direction, as the result of the wavefront distortion induced by transverse density inhomogeneities. Such configurations are typical of the density cavities along the magnetic field encountered in auroral regions where they affect the process of interaction of the Alfvén waves and the plasma, with consequences on the electron acceleration (Génot et al., 1999, 2004). Differently, density enhancements were considered in the description of electron acceleration in open coronal structures (Tsiklauri et al., 2005a,b).

Density channels also play the role of ducts that guide whistler waves in the magnetosheath (Karpman and Kaufman, 1982, 1984; Pasmanik and Trakhtengerts, 2005; Streltsov et al., 2006, 2007, and references therein). Furthermore, quasi-parallel cylindrical structures corresponding to density holes (preferably at large $\beta$ ) or humps (at smaller $\beta$ ), anticorrelated with the local magnetic field intensity and associated with mirror modes (see e.g. Soucek et al., 2008 and references therein) could also produce a lensing effect for Alfvén waves.

The problem then arises of the effect of density inhomogeneities on Alfvén wave filamentation, i.e. in an intermediate regime where dispersion is important and inhomogeneities not strong enough for the usual phase mixing mechanism to dominate. It has been shown in the Hall-MHD framework (Borgogno et al., 2008) that the conditions for the formation of Alfvén filaments can be significantly broadened in the presence of high or low density channels of relatively small amplitude (possibly randomly distributed) aligned with the ambient field. Filaments can even form in situations where the usual filamentation instability is absent. Such enhancements or depressions of the density, by locally affecting the Alfvén velocity, can indeed produce a lensing effect, but an early saturation nevertheless occurs due to diffraction, leading to the formation of filaments with a moderate intensity. Different regimes are to be distinguished, depending on the presence ( $\beta$ typically larger than unity) or absence (small $\beta$ ) of a transverse instability of modulational type for the Alfvén wave in a homogeneous plasma. This transverse instability induces a self-focusing phenomenon whereby nonlinear effects modify the density and the longitudinal fields in a way to reinforce the lensing process. In this case, under the action of a density dip, the resulting Alfvénic structures display temporal oscillations leading to the formation of scales comparable to the ion inertial length. In contrast, in the absence of self-focusing, a relatively weak density hump leads to stationary filaments with a transverse scale prescribed by that of the density hump, that tend to distort and oscillate as the density perturbation is increased.

The Hall-MHD description neglects kinetic effects such as Landau damping, and the question arises whether this effect can inhibit the filament formation. In the case of a homoge- neous plasma, an asymptotic analysis was performed in the limiting case of a pump wavelength large compared with the ion inertial length (Passot and Sulem, 2003c). It turns out that in the case of isotropic temperatures of the ions and the electrons, the filamentation instability requires electrons hotter than ions. Even in this case, the range of values of the parameter $\beta$ for which the instability can occur is strongly limited. Anisotropy of the proton temperatures inhibits even more the instability, while electron temperature anisotropy has a strong destabilizing effect. One aim of the present paper is to extend the analysis of Borgogno et al. (2008), by addressing the influence of kinetic effects on the formation of Alfvén filaments in the presence of density channels. For this purpose, Landau-fluid simulations retaining large-scale low-frequency kinetic effects are presented, together with a hybrid PIC integration of the Vlasov-Maxwell equations. This dual approach, supplemented by a comparison with an asymptotic theory of the filamentation instability in a homogeneous plasma provides a validation of the closure procedure involved in the Landau-fluid model, in regimes where kinetic effects play a significant role. A second goal of this paper is to extend the analysis to situations where the amplitude of the inhomogeneities is larger, allowing the transition to a so-called dispersive phase-mixing regime.

The paper is organized as follows. In Sect. 2, a brief description of the Landau fluid model is presented. Section 3 deals with the linear filamentation instability in a homogeneous plasma, as predicted in the context of a longwavelength weakly nonlinear theory and simulated with the Landau fluid. Section 4 discusses, for different values of the plasma parameters, Landau fluid simulations of the mild Alfvén-wave filamentation that takes place at small $\beta$ in the presence of a high-density channel of moderate amplitude, and provides a comparison with a hybrid PIC simulation in a regime where the small electron to ion temperature ratio makes kinetic effects significant. Section 5 discusses the influence of a density channel of stronger amplitude, leading after the early formation of a magnetic filament to a regime of dispersive phase mixing where, a magnetic structure with the shape of a helical ribbon develops, associated with the onset of strong gradients that permit dissipative effects (not included in the model) to act and heat the plasma. Section 6 summarizes our findings based on a three-dimensional description and discusses their relation with two-dimensional fully kinetic PIC simulations (Tsiklauri et al., 2005a) of the interaction of a circularly polarized Alfvén wave with transverse density perturbations aimed to point out possible mechanisms for electron acceleration.

\section{The Landau-fluid model}

Consider an electrically neutral plasma permeated by a uniform magnetic field, described as a bi-fluid. Neglecting electron inertia, the proton and electron velocities are 
related by $\mathbf{u}_{e}=\mathbf{u}_{p}-\mathbf{j} /(e n)$, where the current is given by $\mathbf{j}=(c / 4 \pi) \nabla \times \mathbf{B}$ and where $n=\rho / m_{p}$ is the number density of the particles and $m_{p}$ the proton mass.

The electrons are viewed as an isotropic isothermal fluid with a (scalar) pressure given by $p_{e}=n T_{e}^{(0)}$, a description also used in the hybrid PIC simulations described below. Differently, the ion pressure is a tensor written as the sum of a gyrotropic part (characterized by the parallel $p_{\| p}$ and perpendicular $p_{\perp p}$ pressures) and of the gyroviscous contribution $\boldsymbol{\Pi}$, in the form $\mathbf{p}_{p}=p_{\perp p} \mathbf{n}+p_{\| p} \boldsymbol{\tau}+\boldsymbol{\Pi}$, with $\mathbf{n}=\mathbf{I}-\widehat{\mathbf{b}} \otimes \widehat{\mathbf{b}}$ and $\boldsymbol{\tau}=\widehat{\mathbf{b}} \otimes \widehat{\mathbf{b}}$, where $\widehat{\mathbf{b}}=\mathbf{B} / B$ is the unit vector along the local magnetic field.

It is convenient to take the ambient field $B_{0}$, the equilibrium density $\rho_{0}$, the Alfvén velocity $v_{A}=B_{0} /\left(4 \pi \rho_{0}\right)^{1 / 2}$ and the ion equilibrium parallel pressure $p_{\| p}^{(0)}$ as units of magnetic field, density, velocity and pressure. The heat fluxes are measured in units of $p_{\| p}^{(0)} v_{A}$. The unit length $L$ is arbitrary and its ratio to the ion inertial length $l_{i}$ (defined as the ratio of the Alfvén velocity and the ion gyrofrequency $\Omega_{p}$ ) denoted by $R=L / l_{i}$. The equations for the plasma density, the proton velocity field and the induction equation then read (the electron pressure contribution in the induction equation being neglected)

$$
\begin{aligned}
\frac{\partial \rho}{\partial t}+ & \nabla \cdot\left(\rho \mathbf{u}_{p}\right)=0 \\
\frac{\partial \mathbf{u}_{p}}{\partial t} & +\mathbf{u}_{p} \cdot \nabla \mathbf{u}_{p}+\frac{\beta_{p}}{2 \rho} \nabla \cdot\left(p_{\perp p} \mathbf{n}+p_{\| p} \boldsymbol{\tau}+\Pi+p_{e} \mathbf{I}\right) \\
& -\frac{1}{\rho}\left[(\mathbf{b} \cdot \nabla) \mathbf{b}-\frac{1}{2} \nabla|\mathbf{b}|^{2}\right]=0 \\
\frac{\partial \mathbf{b}}{\partial t} & =\nabla \times\left(\mathbf{u}_{p} \times \mathbf{b}\right)-\frac{1}{R} \nabla \times\left[\frac{(\nabla \times \mathbf{b}) \times \mathbf{b}}{\rho}\right],
\end{aligned}
$$

where $\beta=8 \pi p_{\| p}^{(0)} / B_{0}^{2}$. Moreover, dropping for simplicity the subscript $p$, the equations for the ion parallel and perpendicular pressures are written in the form

$$
\begin{aligned}
\frac{\partial p_{\perp}}{\partial t} & +\nabla \cdot\left(p_{\perp} \mathbf{u}\right)+p_{\perp} \nabla \cdot \mathbf{u}-p_{\perp} \mathbf{b} \cdot \nabla \mathbf{u} \cdot \mathbf{b} \\
& +\nabla \cdot\left(q_{\perp} \mathbf{b}\right)+q_{\perp} \nabla \cdot \mathbf{b}=0 \\
\frac{\partial p_{\|}}{\partial t} & +\nabla \cdot\left(\mathbf{u} p_{\|}\right)+2 p_{\|} \mathbf{b} \cdot \nabla \mathbf{u} \cdot \mathbf{b} \\
& +\nabla \cdot\left(q_{\|} \mathbf{b}\right)-2 q_{\perp} \nabla \cdot \mathbf{b}=0,
\end{aligned}
$$

where the nongyrotropic contributions of the proton heat fluxes have been neglected. The Landau-fluid closure then consists in expressing the parallel and perpendicular proton heat fluxes $q_{\perp}$ and $q_{\|}$in terms of controlled quantities, in a way consistent with the linear kinetic theory in the longwavelength low-frequency limit near a bi-Maxwellian equilibrium. For this purpose, the various hydrodynamical fields and the nongyrotropic contributions to the pressure and heat transfer are expressed within the above framework in terms of the electromagnetic fluctuations. These expressions involve the phase velocity through the plasma dispersion func- tion (Hazeltine and Waelbroeck, 2004), a quantity not suitable for initial value problems. The closure thus consists in eliminating the plasma dispersion function as often as possible by substitutions in terms of lower moments. In the few terms where it cannot be eliminated, it is replaced by Padé approximants of suitable order, which introduces an Hilbert transform $\mathcal{H}$, physically associated with Landau damping. This leads to the closure equations (Snyder et al., 1997; Passot and Sulem, 2003b)

$$
\begin{aligned}
& \left(\frac{d}{d t}+\frac{\sqrt{\pi \beta_{p}}}{4\left(1-\frac{3 \pi}{8}\right)} \mathcal{H} \partial_{z}\right) q_{\|}=\frac{1}{1-\frac{3 \pi}{8}} \frac{\beta_{p}}{2} \partial_{z}\left(p_{\|}-\rho\right) \\
& \left(\frac{d}{d t}-\frac{\sqrt{\pi \beta_{p}}}{2} \mathcal{H} \partial_{z}\right) q_{\perp}= \\
& \frac{\beta_{p}}{2} \frac{T_{\perp p}^{(0)}}{T_{\| p}^{(0)}} \partial_{z}\left[\left(1-\frac{T_{\perp p}^{(0)}}{T_{\| p}^{(0)}}\right)|b|-\left(\frac{T_{\| p}^{(0)}}{T_{\perp p}^{(0)}} p_{\perp}-\rho\right)\right],
\end{aligned}
$$

where the Hilbert transform of a (scalar) function $f$ is defined as

$\mathcal{H} f(z)=\frac{1}{\pi} V P \int_{-\infty}^{+\infty} \frac{f\left(z^{\prime}\right)}{z-z^{\prime}} d z^{\prime}$

and reduces in Fourier space to the multiplication by $i k_{\|} /\left|k_{\|}\right|$. In Eqs. (6)-(7), the convective derivative $\frac{d}{d t}$ is retained to preserve Galilean invariance.

A simplified form of this closure is obtained by taking the quasi-static limit that leads in Fourier space to the explicit relations

$$
\begin{aligned}
q_{\|}(\mathbf{k}, t)= & -2 \sqrt{\frac{\beta_{p}}{\pi}} \frac{i k_{\|}}{\left|k_{\|}\right|}\left(p_{\|}(\mathbf{k}, t)-\rho(\mathbf{k}, t)\right) \\
q_{\perp}(\mathbf{k}, t)= & \sqrt{\frac{\beta_{p}}{\pi}} \frac{T_{\perp p}^{(0)}}{T_{\| p}^{(0)}} \frac{i k_{\|}}{\left|k_{\|}\right|}\left[\left(1-\frac{T_{\perp p}^{(0)}}{T_{\| p}^{(0)}}\right)|\mathbf{b}(\mathbf{k}, t)|\right. \\
& \left.-\left(\frac{T_{\| p}^{(0)}}{T_{\perp p}^{(0)}} p_{\perp}(\mathbf{k}, t)-\rho(\mathbf{k}, t)\right)\right] .
\end{aligned}
$$

Furthermore, the ion gyroviscous tensor that contributes at the same order as the Hall effect, is approximated to leading order as

$$
\begin{aligned}
& \Pi_{x x}=-\Pi_{y y}=-\frac{p_{\perp}}{2 R}\left(\partial_{y} u_{x}+\partial_{x} u_{y}\right) \\
& \Pi_{x y}=\Pi_{y x}=-\frac{p_{\perp}}{2 R}\left(\partial_{y} u_{y}-\partial_{x} u_{x}\right) \\
& \Pi_{y z}=\Pi_{z y}=\frac{1}{R}\left[2 p_{\|} \partial_{z} u_{x}+p_{\perp}\left(\partial_{x} u_{z}-\partial_{z} u_{x}\right)\right] \\
& \Pi_{x z}=\Pi_{z x}=-\frac{1}{R}\left[2 p_{\|} \partial_{z} u_{y}+p_{\perp}\left(\partial_{y} u_{z}-\partial_{z} u_{y}\right)\right] \\
& \Pi_{z z}=0
\end{aligned}
$$

where the distortion of magnetic field lines has been neglected, an approximation consistent with the linear description of Landau damping. 

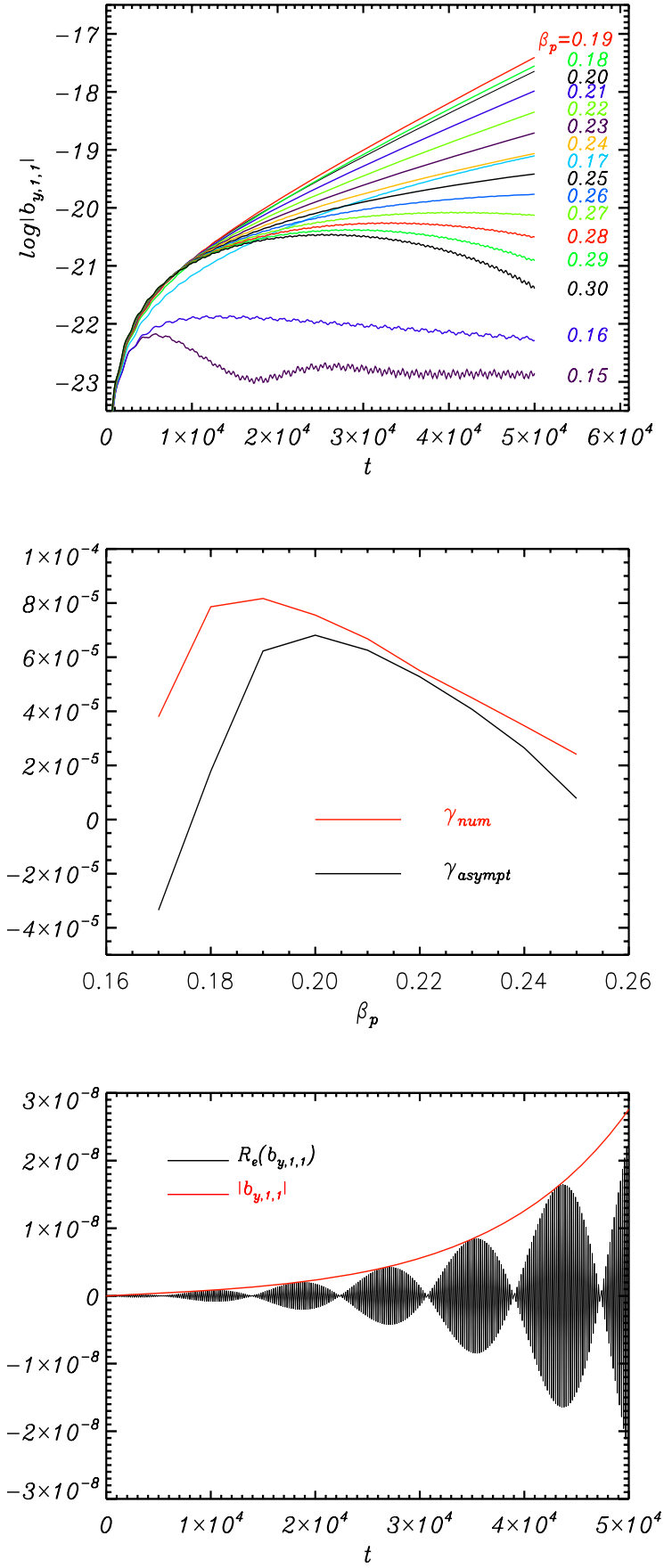

Fig. 1. (top) Time evolution of the linearly most unstable mode of Fourier index $(1,1)$ for the filamentation instability as computed with a two-dimensional Landau-fluid simulation (involving the longitudinal and a transverse direction) at $T_{e}=8 T_{p}$, for a left-hand polarized Alfvén wave with $k l_{i}=0.1$ and an amplitude $\left|b_{\perp 0}\right|=0.05 B_{0}$, assuming various values of $\beta_{p}$ ranging from 0.15 to 0.30 ; (middle) Growth rate of this mode, as measured from the Landau-fluid simulation and estimated from the asymptotic theory; (bottom) Time variation of the amplitude and of the real part of the most unstable mode in the Landau-fluid simulation with $\beta_{p}=0.19$.
More elaborated Landau-fluid models including a refined description of the nongyrotropic contribution, aimed to retain small transverse scales are discussed in Goswami et al. (2005) and Passot and Sulem (2007). The above model is nevertheless sufficient to accurately reproduce the asymptotic dynamics of long-wavelength dispersive waves with a small amplitude compared with the ambient field. Reductive perturbative expansions performed on the Landau-fluid and on the Vlasov Maxwell equations indeed lead to the same multidimensional kinetic derivative nonlinear Schrödinger (KDNLS) equation, up to the replacement of a few nonlocal operators by their Padé approximants (Passot and Sulem, 2003a,b). For the Landau-fluid simulations presented below, no significant difference is observed when using the timedependent closure (Eqs. 6-7) or the static one (Eqs. 9-10).

\section{Filamentation instability in a homogeneous plasma}

An asymptotic theory of the Alfvén wave filamentation instability in a collisionless plasma was discussed in Passot and Sulem (2003c). The analysis is based on a transverse modulation analysis performed on a three-dimensional generalization with mean fields (Passot and Sulem, 2003a) of the kinetic derivative nonlinear Schrödinger equation (Rogister, 1971; Mjølhus and Wyller, 1988), derived from the VlasovMaxwell equations by a long-wavelength reductive perturbative expansion. A main effect of Landau damping is to introduce a diffusive term in the resulting nonlinear Schrödinger equation governing the time evolution of the pump amplitude that, as in the case of the parallel modulational instability, strongly affects the stability/instability ranges predicted in the Hall-MHD framework. Additional parameters such as a significant electron-proton temperature ratio and an electron temperature anisotropy contribute to destabilize a system that is stable in the case of a plasma with isotropic and equal temperatures of the ions and electrons. The analysis of Passot and Sulem (2003c) leads to the conclusion that when a parallel Alfvén wave of wavenumber $k$ and amplitude $\left|b_{\perp 0}\right|$ in an isotropic plasma of density $\rho_{0}$ permeated by an ambient field $B_{0}$, is subject to linear perturbations of wavenumber $K_{\perp}$, the corresponding frequency $\Omega$ (that is possibly complex) is given by

$\Omega=i \frac{v_{A}}{4 k} \nu_{\star} K_{\perp}^{2} \pm v_{A} \sqrt{C \chi_{\star} \frac{\left|b_{\perp 0}\right|^{2}}{B_{0}^{2}} K_{\perp}^{2}+\frac{1}{16 k^{2}} \chi_{\star}^{2} K_{\perp}^{4}}$,

where $v_{A}$ is the Alfvén velocity and $C=(1 / 32)(12+18 \beta+$ $\left.5 \beta^{2}\right) /(1+\beta)$ with $\beta=\beta_{p}\left(1+T_{e} / T_{p}\right)$. The coefficients $v_{\star}$ and $\chi_{\star}$ are functions of $\beta_{p}$ and the temperature ratio, and their computation involves the plasma response functions. They are plotted versus $\beta_{p}$ in Fig. 1b of Passot and Sulem (2003c) in the case of a temperature ratio $T_{e} / T_{p}=8$. It turns out that $v_{\star}$ is always negative. As a consequence, an instability can 
only occur if $\chi_{\star}$ is negative. In this case, the instability rate $\gamma=-i \Omega$ is given by

$\gamma=-\frac{v_{A}}{4 k}\left|v_{\star}\right| K_{\perp}^{2}+v_{A} \sqrt{C\left|\chi_{\star}\right| \frac{\left|b_{\perp 0}\right|^{2}}{B_{0}^{2}} K_{\perp}^{2}-\frac{1}{16 k^{2}} \chi_{\star}^{2} K_{\perp}^{4} .}$

In order to compare the result of this asymptotic theory (that assumes $k l_{i} \sim\left|b_{\perp 0}\right| / B_{0} \ll 1$ ), we performed Landaufluid simulations (with a static description of the heat fluxes), using the same temperature ratio $T_{e} / T_{p}=8$, for a left-hand polarized Alfvén wave with $k l_{i}=0.1$ and $\left|b_{\perp 0}\right| / B_{0}=0.05$. Restricting these simulations to time intervals during which the dynamics can be viewed as linear, a two-dimensional geometry is sufficient. The time evolution of the most unstable modes for different values of $\beta_{p}$ is shown in Fig. 1 (top). As predicted by the theory, the instability is limited to a tiny range of values of $\beta_{p}$ close to 0.2 . The growth rate variation with $\beta_{p}$ is qualitatively similar in the simulation and in the asymptotic theory, up to a shift of about 0.01 (thus a relative discrepancy of about $5 \%$ ) on the position of the instability window and a difference of $1.36 \times 10^{-5}$ (that corresponds to a relative error of about $17 \%$ ) on the maximal growth rate (Fig. 1, middle). Such results can be viewed as consistent with the magnitude of the small parameter that controls the accuracy of the KDNLS asymptotics, that is of order $10 \%$ in the Landau-fluid simulation. A qualitative effect of this deviation from the asymptotic regime is visible on the time evolution of the wave amplitude that is not only amplified as predicted by the theory, but also modulated (Fig. 1, bottom), as would predict a Floquet stability analysis of a monochromatic plane Alfvén wave with a finite wavenumber (Jayanti and Hollweg, 1993).

When the Alfvén wave is taken more dispersive by assuming $k l_{i}=0.4$, the Landau-fluid simulation shows a window of instability in a still limited range $0.14<\beta_{p}<0.22$, with a maximum growth rate $\gamma=1.86 \times 10^{-4}$ for $\beta_{p}=0.16$.

\section{Filamentation in a high-density channel of moderate amplitude}

Alfvén wave filamentation in the presence of density channels, either isolated or distributed in a periodic or random manner was studied in detail in the context of Hall MHD in Borgogno et al. (2008), in the cases of density depressions and enhancements, both for small and moderate values of $\beta$. The aim of the present section is to analyze this phenomenon in the presence of Landau damping. For the sake of simplicity, we concentrate on the effect of a unique channel.

Simulations were first performed in a periodic box with an extension $L_{z}=8 \pi$ in the longitudinal direction and $L_{x}=L_{y}=32 \pi$ in the transverse direction, when measured in units of $L=R l_{i}$ with $R=4$. A resolution of $64^{3}$ collocation points was used. In order to illustrate the influence of a moderate density enhancement, we consider an initial condition consisting in a parallel initial density enhancement of
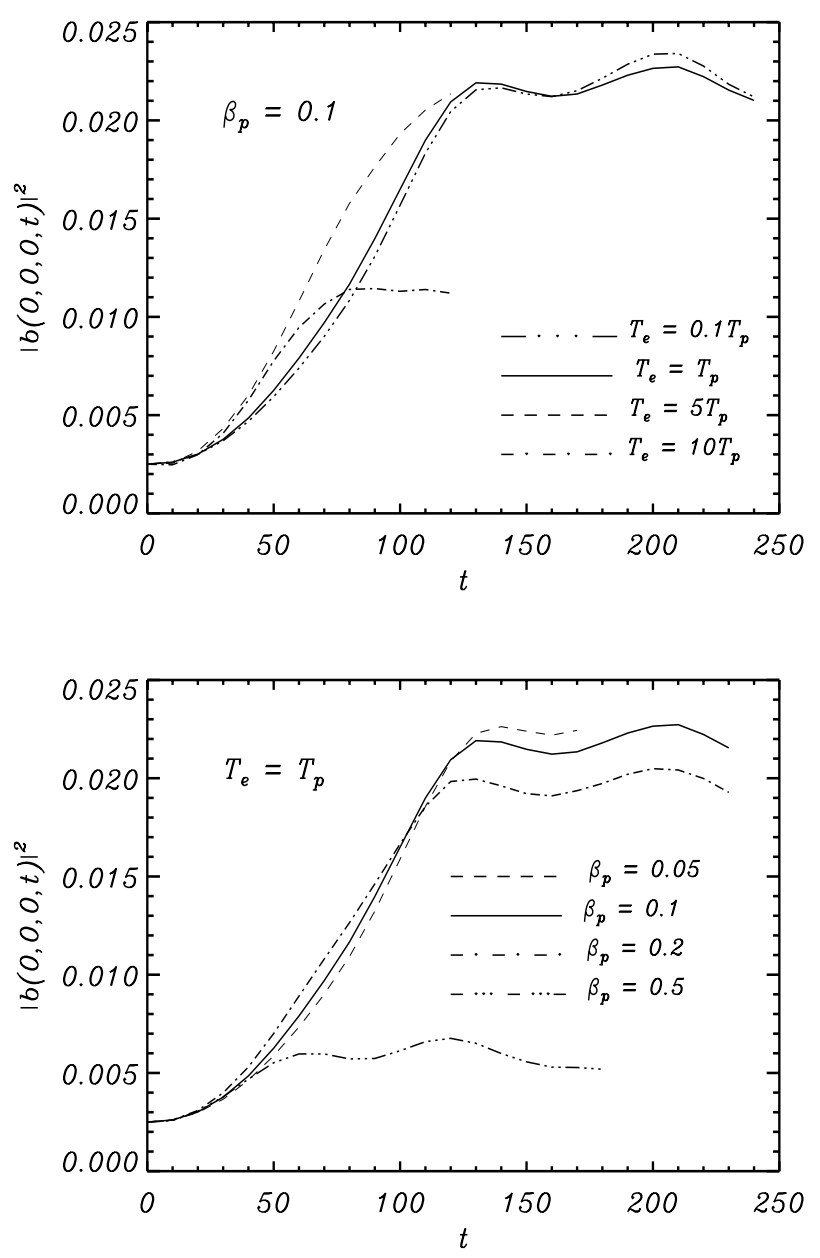

Fig. 2. Time evolution of the maximum intensity of the transverse magnetic field (uniformly located along the $\mathrm{z}$-axis) for $\beta_{p}=0.1$ and various ion-electron temperature ratios (top panel) and for $T_{e}=T_{p}$ and different $\beta_{p}$ (bottom panel). Initial conditions consist in a density enhancement $\rho(x, y, z, t)=1+0.1 \exp \left(-\left(x^{2}+y^{2}\right) / 64\right)$ and a left-hand polarized Alfvén wave of amplitude 0.05 and wavelength equal to $2 \pi$ (in units of 4 ion inertial lengths), propagating parallel to the ambient field.

the form $\rho(x, y, z, t)=1+0.1 \exp \left(-\left(x^{2}+y^{2}\right) / 64\right)$ and a lefthand polarized Alfvén wave with an amplitude of 0.05 and a wavelength $\lambda=2 \pi$. The fluid is initially at rest and the initial ion and electron pressures are isotropic and uniform. Figure 2 displays the time variation of the maximal amplitude of the transverse magnetic field (located on the $\mathrm{x}$-axis) for $\beta_{p}=0.1$ and various temperature ratio $T_{e} / T_{p}$ in the range 0.1 to 10 (upper panel), and for $T_{e} / T_{p}=1$ and different $\beta_{p}$ between 0.05 and 0.5. Another example where the electrons are cooler than the ions is considered below in the context of a comparison with a hybrid PIC simulation. We avoid large $\beta_{p}$ values in order to prevent a strong Landau damping. We see that the intensity (square amplitude) of the wave can be locally amplified by a factor 10 , and then becomes 

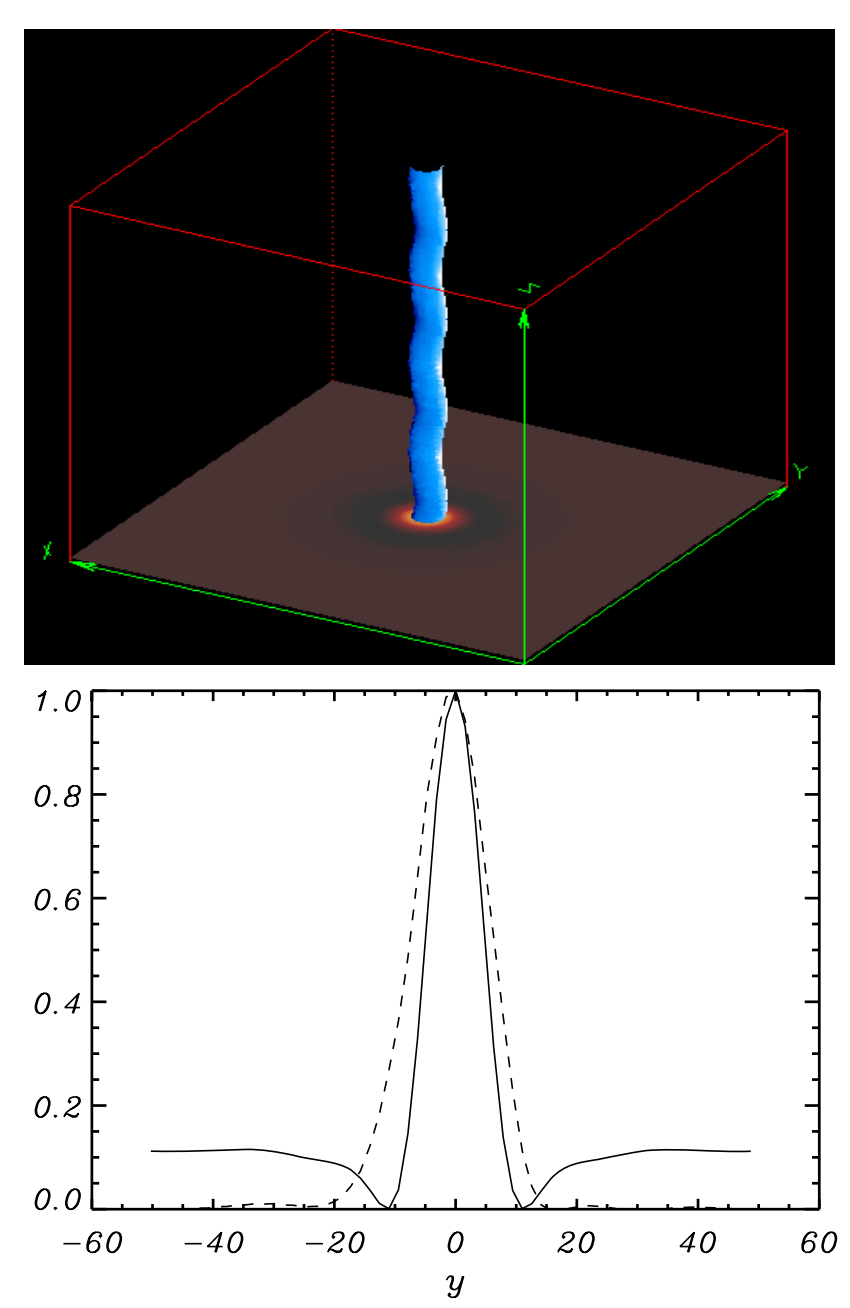

Fig. 3. Top: Alfvén wave filament at $t=220$ in the case $T_{e}=T_{p}$ and $\beta_{p}=0.1$ for the Alfvén wave and the density perturbation considered in Fig. 2. Bottom: in the same conditions, transverse filament profile (solid line) and density perturbation (dashed line), both normalized by their respective maximal value.

quasi-stationary with a slow oscillation. When comparing simulations for different electron-ion temperature ratios, we observe that the maximum intensity of the magnetic filament reaches a limit behavior as the electron temperature is taken comparable to or smaller than that of the ions. The geometry of the resulting structure is essentially always the same and is illustrated in Fig. 3 in the case $\beta_{p}=0.1$ and $T_{e}=T_{p}$, at time $t=220$. We observe the formation of a filament parallel to the ambient field (upper panel). In the lower panel, the profile of this structure along a transverse direction is compared with that of the density channel (both normalized with their maximal value). We observe that in such a simulation, the transverse scale of the filament is prescribed by that of the density channel that has essentially not changed from its initial value. We checked that the energy of the wave (evaluated in the entire computational domain) and the mean parallel and perpendicular temperatures remain almost constant with weak fluctuations whose amplitude does not exceed a relative value of $10^{-3}$. In a different simulation where the diameter of the channel is reduced by a factor 8 , a significantly weaker focusing is obtain. In the case where the density perturbation takes the form of a density depression, the wave is expelled from the density channel both in the case of a low or moderate value of $\beta_{p}$, as observed with $\beta_{p}=0.15$ and $\beta_{p}=1$.

In order to address a regime where the kinetic theory does not reduce to a fluid limit, we also considered a case where $T_{e} / T_{p}=1 / 30$. Since this regime is well adapted to a kinetic description, we performed in this case both Landau fluid and a hybrid PIC simulation with the aim to validate the former approach that retains only part of the kinetic effects. For the latter approach, we used a three-dimensional version of a hybrid code whose algorithm is described in Matthews (1994). We consider a proton-electron plasma where electrons are viewed as a massless, charge neutralizing fluid, with a constant temperature, while the protons, governed by the Vlasov-Maxwell equations are described by a particle-incell model and are advanced by the Boris' implicit scheme that requires the fields to be known at half time steps ahead of the particle velocities, in order to guarantee a better energy conservation. This is achieved by advancing the current density to this time step with only one computational pass through the particle data at each time step. The particle contribution to the current density at the relevant mesh points is evaluated with trilinear weighting followed by smoothing over three points. No smoothing is performed on the electromagnetic fields and, as in the Landau fluid simulations, no resistivity is included in the Ohm's law. The magnetic field is advanced in time with a modified midpoint method, which allows time substepping for the advance of the field. A resolution of 32 points is used in the longitudinal direction and of 128 in the transverse ones, in a computational box of dimensions $L_{z}=32, L_{x}=L_{y}=64$ in units of ion inertial lengths $(R=1)$. The same resolution is used in the Landau-fluid simulation. There are 2048 macroparticles per cell, in order to make the numerical noise as low as possible with the available processor array. The time step for the particle advance is $\Delta t=0.05 / \Omega_{p}$, whereas the magnetic field $\mathbf{B}$ is advanced with a smaller time step $\Delta t_{B}=\Delta t / 4$. In order to reduce the relative importance of the statistical noise in the PIC simulation, we consider an Alfvén wave of amplitude 0.1. The wavelength is taken equal to the longitudinal extension of the computational box. For the ion parallel beta, we used $\beta_{p}=0.3$ and for the electron one $\beta_{e}=0.01$, corresponding to the above mentioned temperature ratio $T_{e} / T_{p}=1 / 30$. The initial density is given by $\rho(0)=1+0.1 \exp \left[-\left(x^{2}+y^{2}\right) / 100\right]$ and, in this case, we assume initial uniform ion and electron temperatures. This initialization is indeed easier to implement in a PIC code than the more physical condition of uniform pressures used in the other simulations presented in this paper. We check that in the PIC simulation the total energy is conserved with an accuracy of $10^{-4}$. 

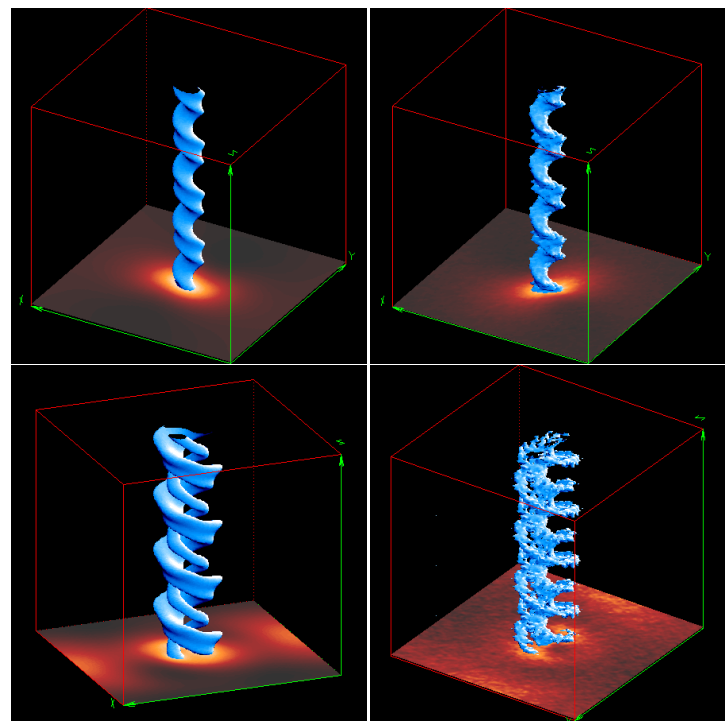

Fig. 4. Isosurfaces of the magnetic filaments observed in the regime $T_{e} / T_{p}=1 / 30$ and $\beta_{p}=0.3$, observed in the Landau fluid (left) and PIC (right) simulations at the respective moments where the structure intensity is maximal (top) and minimal (bottom).

In this kinetic regime, both simulations lead to the formation of magnetic filaments, but the corresponding amplification of the wave intensity is limited to a factor 4 . As previously, we observe in both simulations slow oscillations of the maximum amplitude that are almost periodic. At early time, the agreement between the two computations is very good, but some quantitative difference develops as the formation of the filament proceeds. It concerns for example the maximal amplitude at the moment where the filament is the most intense, PIC simulation then exceeding by about $10 \%$ the Landau-fluid result. In contrast an excellent agreement is obtained at the minimum of the time oscillation and in its neighborhood. The period of the amplitude oscillation is of order of $500 \Omega_{p}^{-1}$ with the Landau fluid and $700 \Omega_{p}^{-1}$ in the PIC simulation. The geometry of the magnetic structures predicted by the two approaches is nevertheless very similar, as shown on Fig. 4 that displays isosurfaces of the transverse magnetic field intensity (chosen at about 3/4 of its instantaneous maximal value), at the respective instants where the intensity of the filament is maximal and minimal. To make the global structure of the filaments more conspicuous, we duplicate by periodicity 4 wavelengths of the pump. Note the helical shape of the filament at high intensity and its replacement by two flattened stripes when the intensity is minimal.

The precise reason of the quantitative difference between the two approaches as the filament intensity is maximal is still unclear, but may have different origins. Kinetic effects not retained by the Landau fluid could be relevant. On the other hand, the initialization of the PIC simulation is delicate, as it is not possible to start with a proper Alfvén mode.

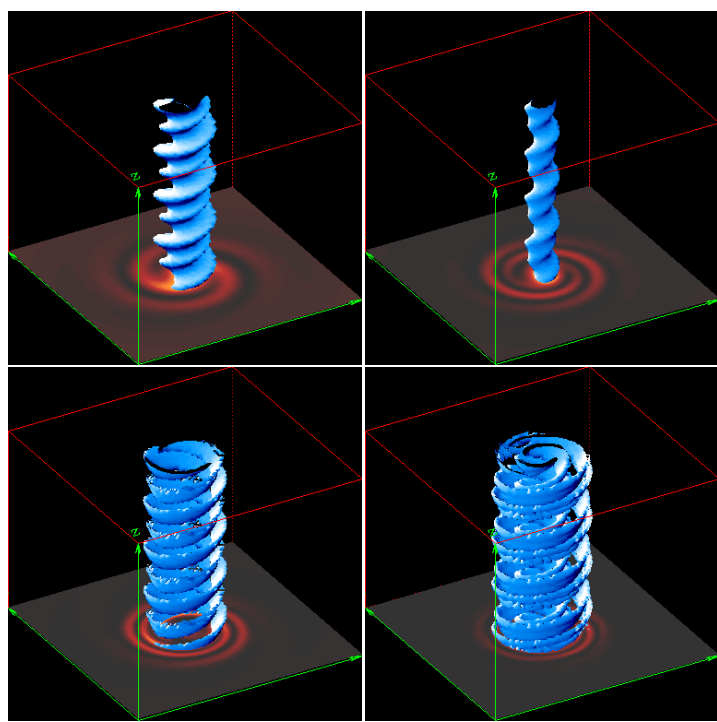

Fig. 5. Magnetic structures developing in the presence of a density perturbation of $50 \%$, described in Sect. 5. From left to right and top to bottom, the various panels correspond to the isosurface of wave intensity of level 0.0130 at time $50,0.0288$ at tome $90,0.00269$ at time 140 and 0.0334 at time 140 .

Indeed, for a perturbation in the electromagnetic field, there is a corresponding complicated perturbation in the distribution function determined by the way the system has been initialized. As a consequence, a counter propagating wave (with right-hand polarization) is launched as well in the simulation, with an amplitude of a few per cent of the intended Alfvén mode. For the considered wave amplitude (10\% of the ambient field), nonlinear effects may thus be non negligible, even at early times.

\section{From filamentation to dispersive phase mixing}

In order to address the Alfvén wave dynamics in the presence of a stronger density enhancement, we first considered an initial density $\rho(x, y, z, t)=1+0.5 \exp \left(-\left(x^{2}+y^{2}\right) / 64\right)$. The parameters of the (uniform) initial pressures and of the Alfvén wave are same as for the run described in Fig. 3 (similar results are in fact obtained when prescribing uniform initial temperatures, as in the comparisons with the PIC simulation). The resolution was however increased to $256^{2} \times 64$ collocation points. A significantly different evolution is observed in Fig. 5 that displays isosurfaces at times $t=50,90$, 140 and 170 . We note that the magnetic filament formed at early time is later on replaced by a structure in the form of a helical ribbon. A more detailed visualization of the transverse geometry is displayed in Fig. 6. While in the case of a small density perturbation discussed in Sect. 4, quasistationary magnetic filaments form with a moderate amplification of the wave intensity and a characteristic transverse 


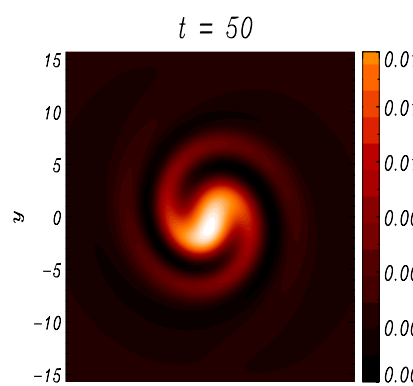

$t=140$
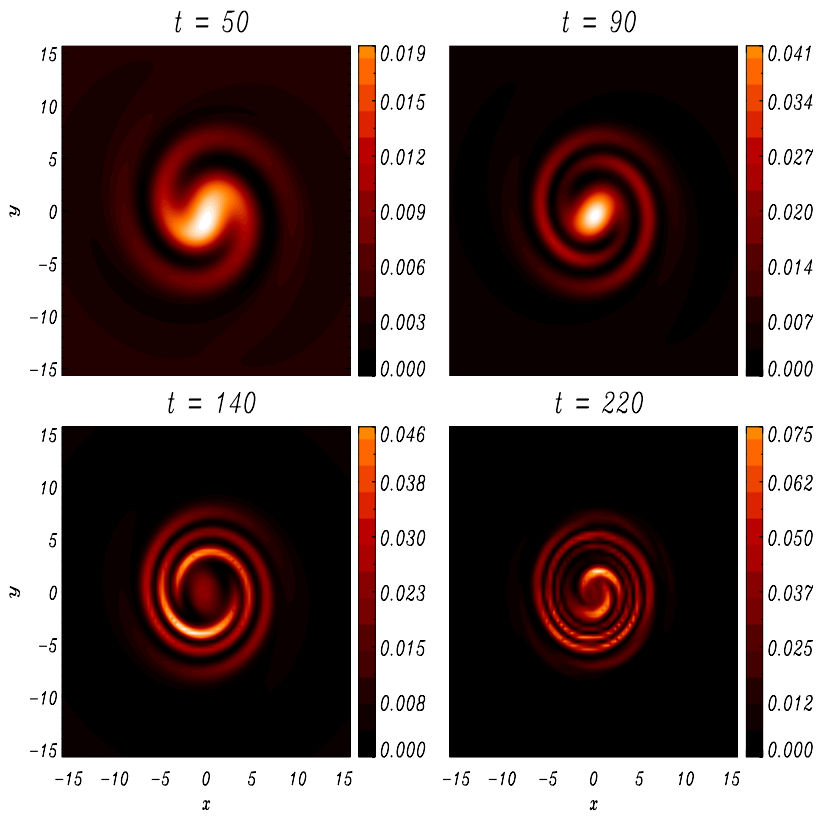

$t=220$

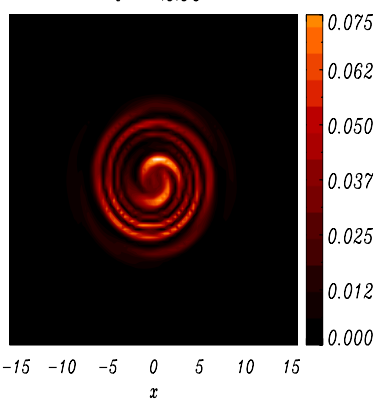

Fig. 6. Color plots of the wave amplitude in the transverse plane $z=0$, in the conditions of Fig. 5 .

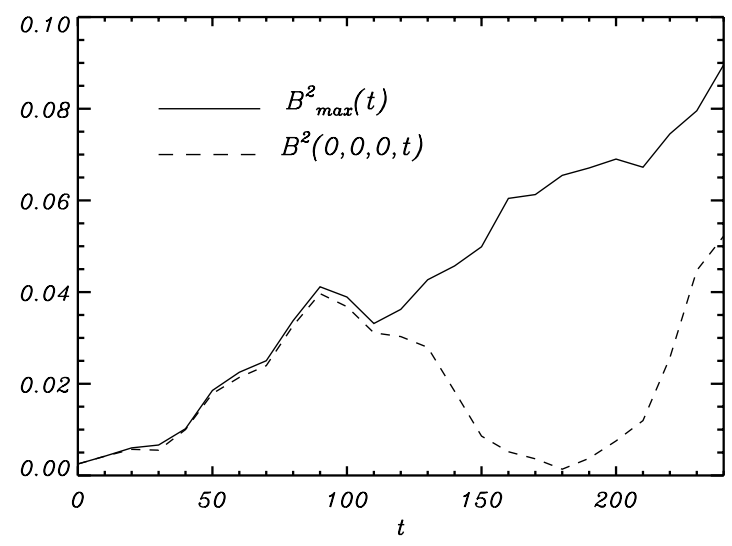

Fig. 7. Variation of the maximum of the wave intensity (solid line) and of its value at the center of the computational box (dashed line) in the conditions of Fig. 5.

scale essentially prescribed by that of the initial density perturbation, in the present regime the dynamics proceeds to the formation of thin magnetic ribbons which strengthen in time and roll up around the central filament whose amplitude oscillate in time. Such an evolution reminds the structures generated by a finite-wavenumber Alfvén wave filamentation in a homogeneous plasma, as exemplified in Fig. 19 of Laveder et al. (2002). Figure 7 displays the temporal evolution of the magnetic intensity at the center of the simulation domain where the filament is located (dashed line) and of its maximum value (solid line). At the end of the simulation, the maximum intensity of the transverse magnetic field reaches

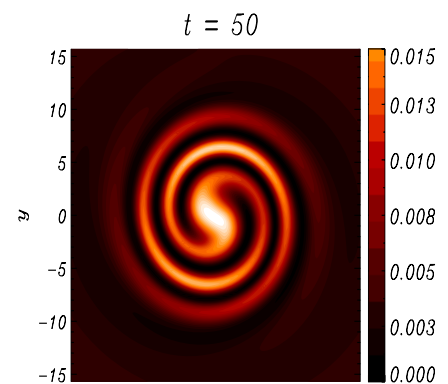

$$
t=120
$$
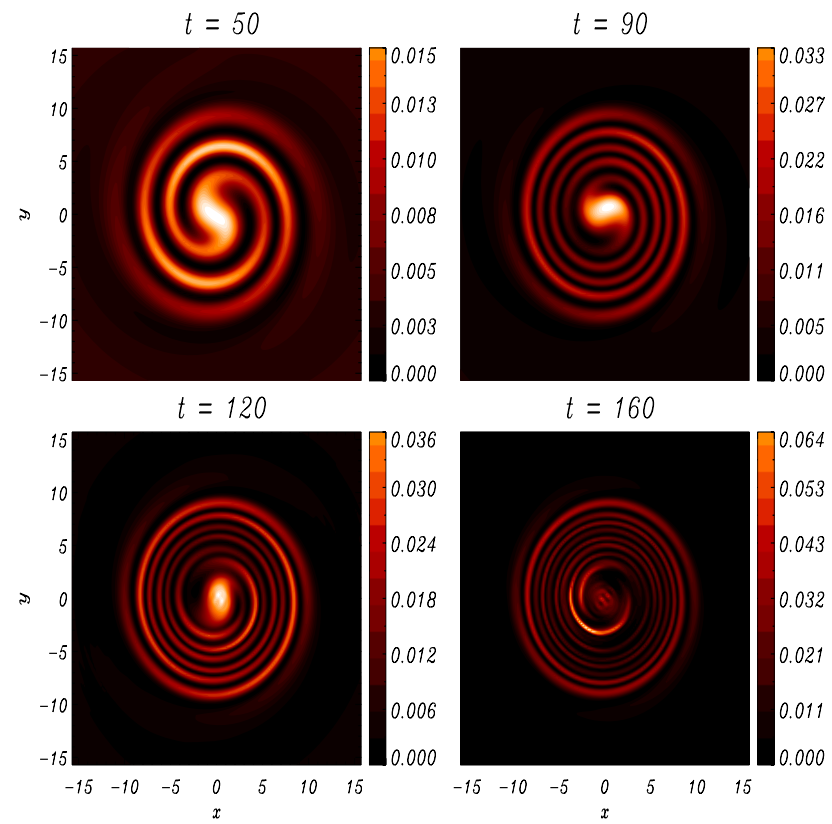

$t=160$

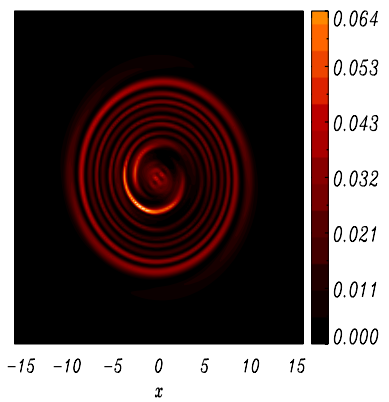

Fig. 8. Color plots of the wave amplitude in the transverse plane $z=0$ for a density channel corresponding to an enhancement of $100 \%$.

0.11 , thus an amplification exceeding a factor 30 . The smallest structures that form are now much smaller than in the previous simulations, with a size that depends on the competition between phase mixing and diffraction effects. Similar geometrical features are visible on the perpendicular and parallel temperature fluctuations, although the latters are weaker than the formers. The mean temperatures are nevertheless not affected, consistent with the purely longitudinal effect of the Landau damping that does not dissipate transverse fluctuations.

Simulations with a density perturbation of $100 \%$ were also performed using a resolution of $512^{2} \times 64$ points. A similar dynamics is obtained, with a more pronounced roll up of the magnetic ribbons associated with the formation of smaller scales (Fig. 8). The simulation was carried on until $t=180$, after which the resolution, although increased in the transverse direction relatively to the $50 \%$ case, becomes insufficient. At this time the maximal amplification of the wave intensity has exceeded a factor 40 . Figure 9 that displays the variation along a transverse direction of the density in the two previous simulations, demonstrates that smaller scales are indeed formed as the amplitude of the initial density channel is increased form 50 to $100 \%$. Since the simulations are interrupted while the maximal magnetic field is still growing because the resolution limits are reached, we conclude that scales even smaller than the ion Larmor radius are generated during the evolution.

Other simulations performed with an Alfvén wave whose wavelength is 10 times shorter show that diffractive effects 
become more important. In this case, a $10 \%$ density channel leads to a rather weak amplification of the magnetic field with the formation of circular rings of wave energy in transverse planes, typical of a diffraction pattern. We thus infer that the required density enhancements necessary for dispersive phase mixing to be efficient are dependent on the Alfvén wavelength (and the plasma parameters) and are more efficient as the ratio between the wavelength and the inertial length is increased.

\section{Concluding remarks}

The present paper concentrates on the formation of magnetic filaments by focusing of a monochromatic Alfvén wave in the presence of a density channel aligned with the ambient magnetic field, and the later transition to a regime of dispersive phase mixing when the initial density enhancement is strong enough. The results are mostly based on the use of a Landau fluid in a formulation valid for transverse scales larger than or comparable to the ion Larmor radius. This model which involves a closure of the fluid hierarchy rigorously established in the limit of linear perturbations about a bi-Maxwellian equilibrium, was also validated by comparison with a hybrid PIC simulation in a strongly nonlinear regime where kinetic effects are significant. We have shown that in the presence of a high-density channel of moderate amplitude in a small-beta plasma, dispersive Alfvén waves can undergo a filamentation process under the effect of a density-induced lensing effect, leading to the formation of magnetic tubes of moderate intensity whose transverse size is prescribed by that of the channel. In contrast, stronger density enhancements can permit a second regime associated with a strong amplification of the maximum of the transverse magnetic field. However, the largest intensities no longer concentrate in a filament but are rather localized in thin ribbon-like structures whose transverse scale is smaller as the amplitude of the initial density channel is increased. For a given density increase, the effect is reduced as the ratio between the pump wavelength and the ion inertial length is decreased. The above evolution contrasts with the usual phase mixing mechanism in non-dispersive MHD where the strong gradients that form are concentrated on rather localized oblique shocks.

Two-dimensional fully kinetic PIC simulations (using a modest ion to electron mass ratio $m_{i} / m_{e}=16$ ) performed by Tsiklauri et al. (2005a,b) and Mottez et al. (2006) with a density channel corresponding to an enhancement of several hundreds of per cent, lead to the development of fluctuations at scales of the order of the electron Larmor radius, thus permitting electron acceleration. Our results suggest that retaining the three-dimensional geometry can be important as it leads to the formation of strong gradients on extended spatial regions in the form of thin helical ribbons spiraling around a central tube. A detailed investigation of the resulting heating
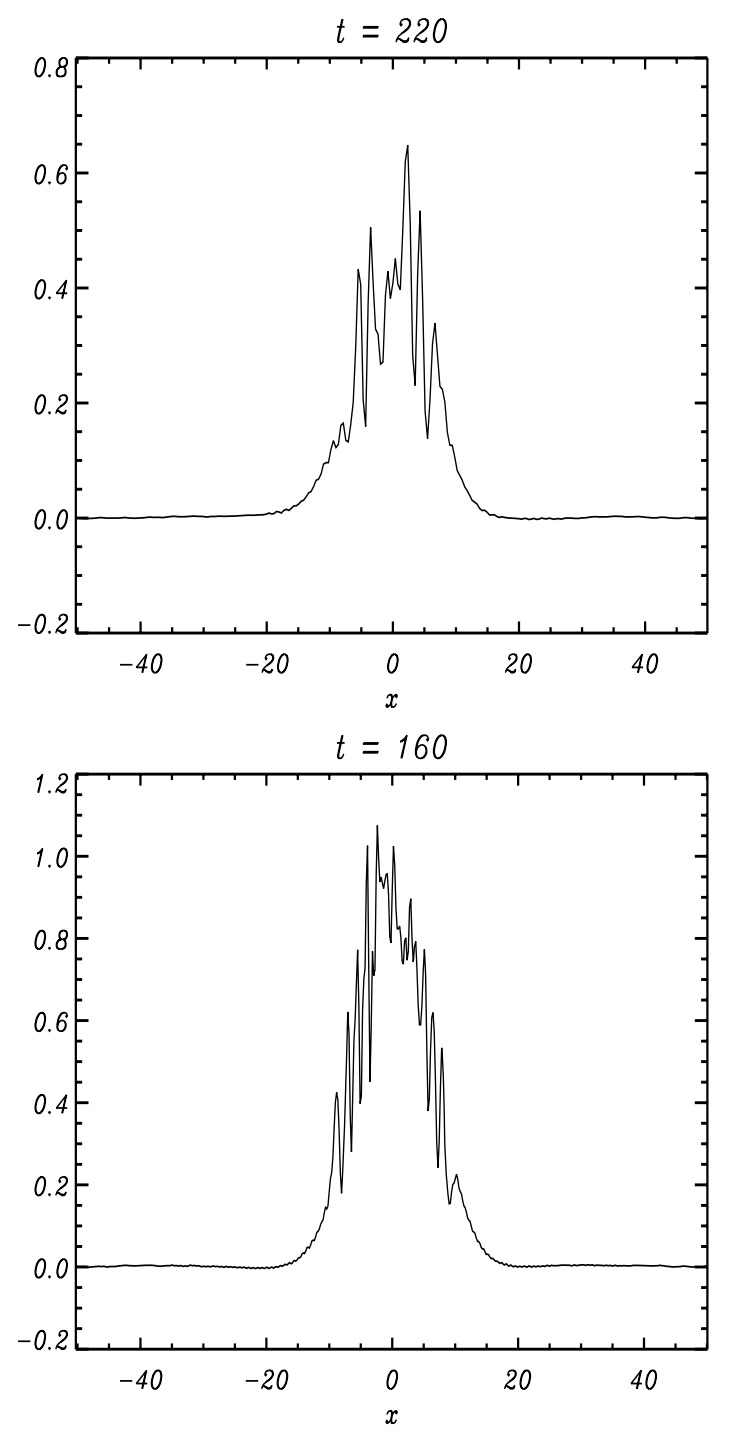

Fig. 9. Profile in a transverse direction of the density fluctuations $\rho-1$ at the end of the simulation for a density channel of amplitude $50 \%$ (top) and of $100 \%$ (bottom).

and particle acceleration is necessary. It requires the inclusion of a kinetic description of the electron dynamics. In this context, a parametric investigation of dispersive phase mixing in terms of plasma parameters would be most useful, but is beyond the scope of the present study.

Acknowledgements. This work benefited of support from the Barrande-Egide project 14226SH, the French Ministère de l'Enseignement Supérieur et de la Recherche, and the CNRS programs "Soleil-Terre". PH and PMT acknowledge the Czech grant GAAV IAA300420702. Landau-fluid simulations were performed on the "Mésocentre SIGAMM" machine hosted by Observatoire de la Côte d'Azur, and the PIC simulations on Amalka supercomputing facility at IAP, ASCR. 
Edited by: R. Pottelette

Reviewed by: two anonymous Referees

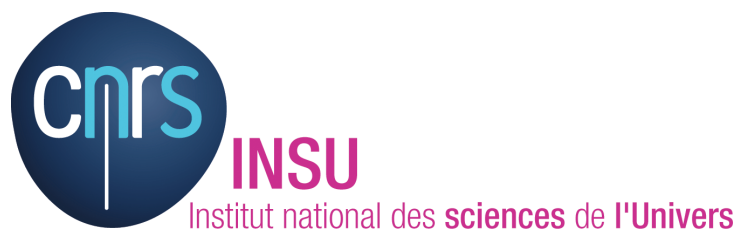

The publication of this article is financed by CNRS-INSU.

\section{References}

Alexandrova, O., Mangeney, A., Maksimovic, M., Lacombe, C., Cornilleau-Wehrlin, N., Lucek, E. A., Décréau, P. M. E., Bosqued, J.-M., Travnicek, P., and Fazakerley, A. N.: Cluster observations of finite-amplitude Alfvén waves and small-scale magnetic filaments downstream of a quasi-perpendicular shock, J. Geophys. Res., 108, A05207, doi:10.1029/2003JA010056, 2004.

Alexandrova, O., Mangeney, A., Maksimovic, M., CornilleauWehrlin, N., Bosqued, J.-M., and André, M.: Alfvén vortex filaments observed in magnetosheath downstream of a quasiperpendicular bow shock, J. Geophys. Res., 111, A12208, doi: 10.1029/2006JA011934, 2006.

Borgogno, D., Laveder, D., Passot, T., Sulem, C., and Sulem, P. L.: Filamentation of dispersive Alfvén waves in density channels: Hall-MHD description, Phys. Plasmas, 15, 062302, doi:10.1063/1.2930470, 2008.

Buti, B.: Nonlinear Alfvén waves in inhomogeneous plasmas, Geophys. Res. Lett., 18, 809-912, 1991.

Buti, B., Galinski, V. L., Shevchenko, V. I., Lakhina, G. S., Tsurutani, B. T., Golstein, B. E., Diamond, P., and Medvedev, V. M.: Evolution of Nonlinear Alfvén Waves in Streaming Inhomogeneous Plasmas, Astrophys. J., 523, 849-854, 1999.

Champeaux, S., Passot, T., and Sulem, P. L.: Alfvén wave filamentation, J. Plasma Phys., 58, 665-690, 1997.

Dreher, J., Laveder, D., Grauer, R., Passot, T., and Sulem, P. L.: Formation and disruption of Alfvénic filaments in Hall-magnetohydrodynamics, Phys. Plasmas, 12, 052319, doi:10.1063/1.1897717, 2005.

Génot, V., Louarn, P., and Quéau, D. L.: A study of the propagation of Alfvén waves in the auroral density cavities, J. Geophys. Res., 104, 22643-22656, 1999.

Génot, V., Louarn, P., and Mottez, F.: Alfvén wave interaction with inhomogeneous plasmas: acceleration and energy cascade towards small-scales, Ann. Geophys., 22, 2081-2096, 2004 , http://www.ann-geophys.net/22/2081/2004/.

Goswami, P., Passot, T., and Sulem, P. L.: A Landau fluid model for warm collisonless plasmas, Phys. Plasmas, 12, 102109, 10.1063/1.2096582, 2005.

Hazeltine, R. and Waelbroeck, F.: The Framework of Plasma Physics, Frontiers in Physics, Westview Press, 2004.

Heyvaerts, J. and Priest, E. R.: Coronal heating by phase-mixed shear Alfvén waves, Astron. Astrophys., 117, 220-234, 1983.

Ireland, J. and Priest, E. R.: Phase-mixing in dissipative Alfvén waves, Solar Phys., 173, 31-51, 1997.
Jayanti, V. and Hollweg, J.: On the dispersion relations for parametric instabilities of parallel-propagating Alfvén waves, J. Geophys. Res., 98, 13247, 1993.

Karpman, V. I. and Kaufman, R. N.: Whistler wave propagation in density ducts, J. Plasma Phys., 27, 225-238, 1982.

Karpman, V. I. and Kaufman, R. N.: Whistler wave-propagation in magnetospheric ducts (in the equatorial region), Planet. Space, 32, 1505-1511, 1984.

Laveder, D., Passot, T., and Sulem, P. L.: Transverse dynamics of dispersive Alfvén waves: I. Direct numerical evidence of filamentation, Phys. Plasmas, 9, 293-304, 2002.

Laveder, D., Passot, T., Sulem, C., Sulem, P. L., Wang, D., and Wang, X. P.: Wave collapse in dispersive magnetohydrodynamics: direct simulations and envelope modeling, Physica D., 184, 237-258, 2003.

Matthews, A.: Current advance method and cyclic leapfrog for 2D multispecies hybrid plasma simulations, J. Comput. Phys., 112, 102-116, 1994.

Mjølhus, E. and Wyller, J.: Nonlinear Alfvén wave in a finete-beta plasma, J. Plasma Phys., 40, 299-319, 1988.

Mottez, F., Génot, V., and Louarn, P.: Comment on "PIC simulations of circularly polarised Alfvén wave phase mixing: a new mechanism for electron acceleration in collisionless plasmas", Astron. Astrophys., 449, 449-450, 2006.

Pasmanik, D. L. and Trakhtengerts, V. Y.: Dispersion properties of ducted whistlers, generated by lightning discharge, Ann. Geophys., 23, 1433-1439, 2005, http://www.ann-geophys.net/23/1433/2005/.

Passot, T. and Sulem, P. L.: Long-Alfvén-wave trains in collisionless plasmas. I. Kinetic theory, Phys. Plasmas, 10, 3887-3905, 2003a.

Passot, T. and Sulem, P. L.: Long-Alfvén-wave trains in collisionless plasmas. II. A Landau-fluid approach, Phys. Plasmas, 10, 3906-3913, 2003b.

Passot, T. and Sulem, P. L.: Filamentation instability of long Alfvén waves in warm collisionless plasmas, Phys. Plasmas, 10, 3914 3921, 2003c.

Passot, T. and Sulem, P. L.: Collisionless magnetohydrodynamics with gyrokinetic effects, Phys. Plasmas, 14, 082502, doi:10.1063/1.2751601, 2007.

Rogister, A.: Parallel Propagation of Nonlinear Low-Frequency Waves in High- $\beta$ Plasma, Phys. Plasmas, 9, 2733-2739, 1971.

Ruderman, M. S.: Propagation of solitons of the derivative nonlinear Schrödinger equation in a plasma with fluctuating density, Phys. Plasmas, 9, 2940-2945, 2002.

Shukla, P. K. and Stenflo, L.: Filamentation instability of Alfvén waves, Astrophys. Space Sci., 155, 145-147, 1989.

Snyder, P. B., Hammett, G. W., and Dorland, W.: Landau fluid models of collisionless magnetohydrodynamics, Phys. Plasmas, 4, 3974-3985, 1997.

Soucek, J., Lucek, E., and Dandouras, I.: Properties of magnetosheath mirror modes observed by Cluster and their responses to changes in plasma parameters, J. Geophys. Res., 113, A04203, doi:10.1029/2007JA012649, 2008.

Streltsov, A. V., Lampe, M., Manheimer, W., Ganguli, G., and Joyce, G.: Whistler propagation in inhomogeneous plasma, J. Geophys. Res., 111, A03216, doi:10.1029/2005JA011357, 2006.

Streltsov, A. V., Lampe, M., and Ganguli, G.: Whistler propagation in nonsymmetrical density channels, J. Geophys. Res., 112, 
A06626, doi:10.1029/2006JA012093, 2007.

Sulem, C. and Sulem, P. L.: The Nonlinear Schrödinger equation: Self-focusing and wave collapse, vol. 139 of Aplied Mathematical Sciences, Springer, 1999.

Tsiklauri, D., Sakai, J. I., and Saito, S.: Particle-in-cell simulations of circularly polarised Alfvén wave phase mixing: a new mechanism for electron acceleration in collisionless plasmas, Astron. Astrophys., 435, 1105-1113, 2005a.
Tsiklauri, D., Sakai, J. I., and Saito, S.: Phase mixing of shear Alfvén waves as a new mechanism for electron acceleration in collisionless, kinetic plasmas, New J. Phys., 7, 79, 2005 b. 